Ian Carter-O'Connell

Santa Clara University

Department of Chemistry and Biochemistry

500 El Camino Real

Santa Clara, CA 95053, United States

Phone: 408-554-5033

E-mail: icarteroconnell@scu.edu

\title{
Rapid Analysis of ADP-Ribosylation Dynamics and Site-Specificity Using TLC-MALDI
}

Sean R. Wallaceł, Leila Y. Chihabł, Miles Yamasaki, Braden T. Yoshinaga, Yazmin M. Torres, Damon

Rideaux, Zeeshan Javed, Soumya Turumella, Michelle Zhang, Dylan R. Lawton, Amelia A. Fuller, and lan Carter-O'Connell*

Santa Clara University, Department of Chemistry and Biochemistry, Santa Clara, California, 95053, United States.

${ }^{*}$ corresponding author 


\section{Supporting Information}

Protein and Peptide Preparation. Recombinant proteins (P14 and P15) were preceded by a 6x His tag and were expressed and purified as previously described. ${ }^{1}$ The P14i1 and P14i3 peptides were obtained from GenScript.

P14i2 Peptide Synthesis and Purification. The P14i2 peptide was prepared on a $0.1 \mathrm{mmol}$ scale on a CEM Liberty Blue peptide synthesizer using the Fmoc/Ot-Bu protecting scheme as previously described. ${ }^{2}$ Crude $^{2}$ peptides were purified to $>95 \%$ by reverse-phase high-performance liquid chromatography (RP-HPLC) on a Hitachi Chromaster 5000 instrument equipped with a semi-preparative AAPPTec Spirit C18 column (5 mM, $10.0 \mathrm{~mm} \times 25 \mathrm{~cm}$ ) using a linear gradient of $10 \%-60 \%$ methanol (solvent B) in $0.1 \%$ aqueous TFA (solvent A) at a flow rate of $3 \mathrm{~mL} / \mathrm{min}$. Peaks eluted were detected by absorbance at $220 \mathrm{~nm}$. All data were visualized with EZChrom software (Agilent). Purified peptides were lyophilized to afford white powders. The molar masses of purified peptides were confirmed by electrospray mass spectrometry in positive ion mode using a Thermo LCQ Fleet mass spectrometer (ThermoFisher).

ADP-Ribosylation Assay Clean-up. Protein desalting was performed via buffer exchange with $490 \mu \mathrm{L}$ of $0.1 \%$ TFA, thrice, targeting a final retentate volume of $\leq 5 \mu \mathrm{L}$. Desalted samples were immediately mixed in a 1:1 ratio with a matrix solution consisting of $5 \mathrm{mg} \mathrm{mL}^{-1}$ Super-DHB (Sigma-Aldrich), $5 \mathrm{mg} \mathrm{mL}^{-1} \mathrm{\alpha}-\mathrm{CHCA}, 50 \%$ acetonitrile, and $0.05 \%$ TFA and stored on ice. For the Western blot controls, $2 \mu \mathrm{L}$ of the reaction mixture was mixed with $2 \mu \mathrm{L}$ of $2 x$ sample buffer and substrate labeling was detected via immunoblot using the pan-ADPribose detection reagent MABE1016 (Millipore) and quantified using ImageLab v5.2 (Bio-Rad) and compared against the total substrate load as determined using Ponceau $S$ stain. Peptide samples were desalted using ZORBAX SB-C18 peptide cleanup pipette tips (Agilent). Desalted peptide samples were immediately mixed in a $1: 1$ ratio with a matrix solution consisting of $10 \mathrm{mg} \mathrm{mL}^{-1} \mathrm{\alpha}-\mathrm{CHCA}$ (Sigma-Aldrich), $50 \%$ acetonitrile, and $0.05 \%$ TFA and stored on ice.

TLC-MALDI Preparation and Optimization. Based on previous reports, ${ }^{3}$ we tested CHCA, DHB, and sinapic acid (SA) either alone or in combination as initial thin-layers. In each case - with both peptides and proteins we observed higher intensities and better resolution in our spectra when CHCA was used alone (data not shown). $1 \mu \mathrm{L}$ of the desalted sample mix in matrix solution was deposited on the $\alpha$-CHCA layer and left to dry to completion. Calibrations were performed using either the ProteoMass Peptide or ProteoMass Protein calibration kits (Sigma-Aldrich) per the manufacturer's instructions. MALDI-TOF experiments were performed using a MALDI-8020 instrument (Shimadzu).

MS Acquisition Parameters and Data Analysis. For collection of peptide spectra the mass range was set to $1500-4000 \mathrm{Da}$. 50 laser shots were fired for each profile at a frequency of $50 \mathrm{~Hz}$ and 2500 total shots were collected per sample. Post-acquisition baseline subtraction and smoothing was performed using MALDI Solutions (Shimadzu) with the following parameters: baseline filter width set to 10, Gaussian smoothing with a smoothing width of 10 . For collection of protein spectra the mass range was set to $30000-50000 \mathrm{Da}$. 100 laser shots were fired for each profile at a frequency of $50 \mathrm{~Hz}$ and 2500 total shots were collected per sample. Pulse extraction was used for both P14 and P15 at 29043 and 26455 Da, respectively (2/3rds of the largest observed mass). Post-acquisition baseline subtraction and smoothing was performed with the following parameters for protein samples: baseline filter width set to 450, Savitsky-Golay smoothing with a smoothing width of 150. Following data acquisition, spectral intensities were min-max normalized between 0.0 and 1.0 in Origin 2020 (OriginLab) prior to import in Fityk. ${ }^{4}$ Peak fitting was performed in Fityk using the automated peakpicking algorithm with standard Gaussian distributions. For each experiment, the half-width, half-max values for each ADPr-modified peak were equalized. The center of each peak was recorded and spectra were rejected if any observed mass differed from the predicted mass by more than $1 \%$. The peak fits were integrated to determine the area under the curve and the resulting values were utilized to calculate the relative levels of ADP-ribosylation. Student's $t$-tests were performed with a two-tailed distribution to determine significance for any observed differences between experimental conditions in Excel (Microsoft). 
Identification of Solvent Exposed D/E Residues. Crystal structures of the PARP15 catalytic domain (3BLJ)5, the yeast SUMO1 domain $(3 \mathrm{QHT})^{6}$, and the AlphaFold prediction of PARP14 ${ }^{7}$ were analyzed for solvent accessible surface areas using the GETAREA plugin. ${ }^{8}$ Residues that had a ratio of side-chain surface area that was greater than $50 \%$ as compared to random coil surface area were considered outward facing residues.

\section{Supporting References}

(1) Carter-O'Connell, I.; Jin, H.; Morgan, R. K.; David, L. L.; Cohen, M. S. Engineering the Substrate Specificity of ADP-Ribosyltransferases for Identifying Direct Protein Targets. J. Am. Chem. Soc. 2014, 136 (14), 5201-5204. https://doi.org/10.1021/ja412897a.

(2) Miller, S. E.; Yamada, Y.; Patel, N.; Suárez, E.; Andrews, C.; Tau, S.; Luke, B. T.; Cachau, R. E.; Schneider, J. P. Electrostatically Driven Guanidinium Interaction Domains That Control HydrogelMediated Protein Delivery In Vivo. ACS Cent. Sci. 2019, 5 (11), 1750-1759. https://doi.org/10.1021/acscentsci.9b00501.

(3) Signor, L.; Boeri Erba, E. Matrix-Assisted Laser Desorption/lonization Time of Flight (MALDI-TOF) Mass Spectrometric Analysis of Intact Proteins Larger than 100 KDa. J. Vis. Exp. JoVE 2013, No. 79, 50635. https://doi.org/10.3791/50635.

(4) Wojdyr, M. Fityk: A General-Purpose Peak Fitting Program. J. Appl. Crystallogr. 2010, 43 (5), $1126-1128$. https://doi.org/10.1107/S0021889810030499.

(5) Karlberg, T.; Klepsch, M.; Thorsell, A.-G.; Andersson, C. D.; Linusson, A.; Schüler, H. Structural Basis for Lack of ADP-Ribosyltransferase Activity in Poly(ADP-Ribose) Polymerase-13/Zinc Finger Antiviral Protein. J. Biol. Chem. 2015, 290 (12), 7336-7344. https://doi.org/10.1074/jbc.M114.630160.

(6) Gilbreth, R. N.; Truong, K.; Madu, I.; Koide, A.; Wojcik, J. B.; Li, N.-S.; Piccirilli, J. A.; Chen, Y.; Koide, S. Isoform-Specific Monobody Inhibitors of Small Ubiquitin-Related Modifiers Engineered Using StructureGuided Library Design. Proc. Natl. Acad. Sci. U. S. A. 2011, 108 (19), 7751-7756. https://doi.org/10.1073/pnas.1102294108.

(7) Jumper, J.; Evans, R.; Pritzel, A.; Green, T.; Figurnov, M.; Ronneberger, O.; Tunyasuvunakool, K.; Bates, R.; Žídek, A.; Potapenko, A.; et al. Highly Accurate Protein Structure Prediction with AlphaFold. Nature 2021, 596 (7873), 583-589. https://doi.org/10.1038/s41586-021-03819-2.

(8) Fraczkiewicz, R.; Braun, W. Exact and efficient analytical calculation of the accessible surface areas and their gradients for macromolecules. J. Comput. Chem. 1998, 19 (3), 319-333.

https://doi.org/10.1002/(SICI)1096-987X(199802)19:3<319::AID-JCC6>3.0.CO;2-W. 


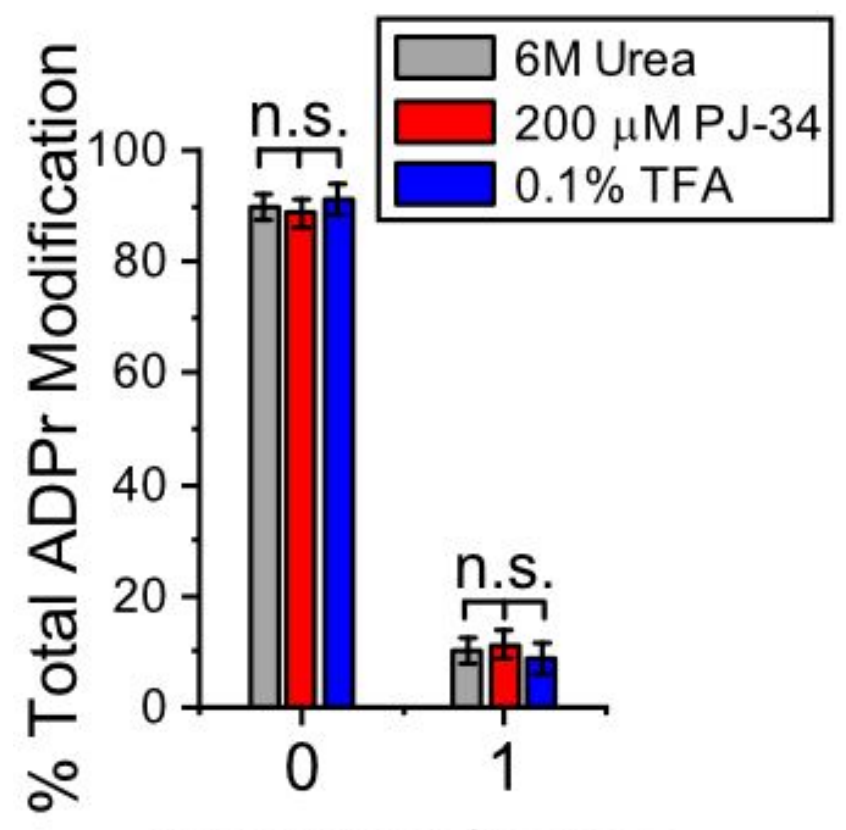

\section{ADPr Modification}

Figure S1. Choice of quenching agent does not impact non-enzymatic ADP-ribose removal. P14 was incubated in the presence of $100 \mu \mathrm{M} \mathrm{NAD}^{+}$and subjected to TLC-MALDI using the listed quenching agents. The bar graph depicts the fraction of the total protein that has been modified at 0 or 1 distinct sites (mean \pm S.E.M., $n=3$ ). N.S. represents no statistical difference, two-tailed Student's $t$ test.

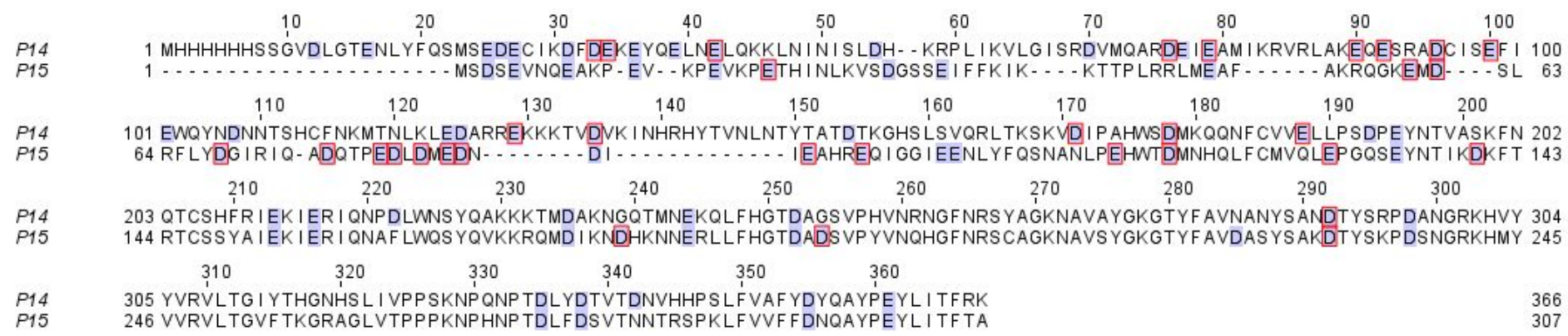

Figure S2. Sequence alignment of the P14 and P15 constructs. The acidic residues E and D are highlighted in blue. Residues that were identified as solvent exposed are highlighted in red.
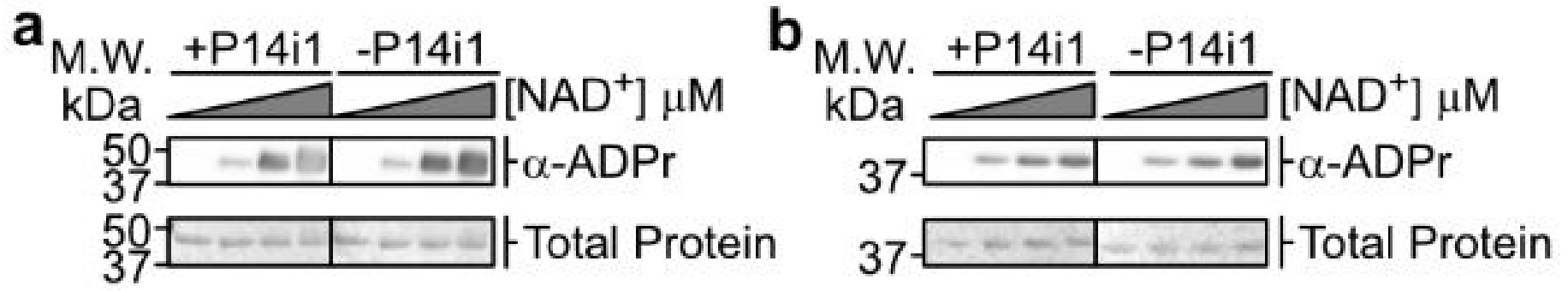

Figure S3. P14 and P15 activity is unaffected by the presence of P14i1. (a) P14 was subjected to automodification in the presence and absence of P14i1 and the blot was first imaged using a Ponceau $S$ stain to detect total protein (bottom image) and then immunoblotted with MAB1016 to detect substrate ADPr (top image). (b) P15 was treated as in (a). 
a

M.W.

$\mathrm{kDa} \circ \bullet \mathrm{NAD}^{+}$

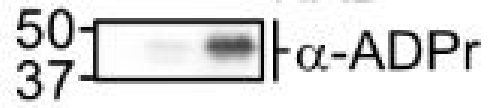

$30-7$ Total Protein b

M.W. • ○ HA

$\mathrm{kDa} \circ \bullet \bullet \mathrm{NAD}^{+}$

$37-\square$ $\alpha-\mathrm{ADPr}$

$37 \square$ Total Protein

Figure S4. In vitro HA ADPr removal assay. (a) Auto-modified P14 was subjected to HA treatment and the resulting blot was first imaged using a Ponceau $S$ stain to detect total protein (bottom image) and then immunoblotted with MAB1016 to detect substrate ADPr (top image). (b) Auto-modified P15 was treated as in (a). Closed circles represent the addition of either HA or NAD ${ }^{+}$. 